\title{
Association between tooth loss and risk of oesophageal cancer: a dose-response meta-analysis
}

\author{
Yadong Wang ${ }^{1 \dagger}$, Juxiang Peng ${ }^{2 \dagger}$, Yan Li ${ }^{1}$, Hong Luo ${ }^{1}$, Guanglei Huang ${ }^{1}$, Siyang Luo ${ }^{1}$, Xinhai Yin ${ }^{1}$ \\ and Jukun Song ${ }^{1 *}$
}

\begin{abstract}
Many epidemiological studies have found that tooth loss is associated with susceptibility to oesophageal cancer. However, a definitive answer is yet to be discovered, and the findings are inconclusive. We performed a meta-analysis to assess the relationship between tooth loss and oesophageal cancer risk. We searched PubMed and Embase databases to screen eligible studies up to June 2015. Nine observational studies (eight articles) involving 2604 patients and 113,995 participants were included in the meta-analysis. The combined odds ratio for tooth loss and oesophageal cancer was 1.53 (95\% Cl 1.02-2.29) for the high versus lowest teeth loss categories. However, inconsistent results were detected in the stratified and sensitivity analysis. In dose-response analysis, the summary odds ratio for each one tooth loss increment was 1.01 (95\% Cl 1.00-1.02). The current evidence, based solely on six case-control studies and three cohort studies, suggests that tooth loss is a potential marker of oesophageal cancer. However, no firm conclusion can be drawn at this time that tooth loss may play a causal role in development of oesophageal cancer. Additional large-scale and high-quality prospective studies are required to evaluate the association between tooth loss and risk of oesophageal cancer.
\end{abstract}

\section{Background}

Oesophageal cancer, including squamous cell carcinoma and adenocarcinoma, is the seventh most common leading cause of cancer-related death in males in the United States, with an estimated 12,720 deaths in 2016, and one of the most common incident cancer, with an estimated 13,460 new cases (Siegel et al. 2016). Oesophageal cancer has been estimated to become a major concern with the rising trend of incidence in adult population. In each year, more than 450,000 people worldwide are diagnosed with the oesophageal cancer (Pennathur et al. 2013). The mortality from these cancers is high because most of the oesophageal cancer cases reported have been advanced at diagnosis (Napier et al. 2014). Therefore, finding and preventing the risk factors are important and significant

\footnotetext{
*Correspondence: songjukun@163.com

${ }^{\dagger}$ Yadong Wang and Juxiang Peng contributed equally to this work

${ }^{1}$ Department of Oral and Maxillofacial Surgery, Guizhou Provincial People's Hospital, Guizhou 550002, China

Full list of author information is available at the end of the article
}

in research. In the past decades, established risk factors for oesophageal cancer, including smoking tobacco, heavy alcohol drinking, poor diet (low fresh fruit and vegetable intake) and low socioeconomic status collectively account for less than half of all oesophageal cancer cases (Castellsague and Munoz 1997; Engel et al. 2003; Enzinger and Mayer 2003; Peng et al. 2016; Wang et al. 2012). The above mentioned data highlight the importance of screening patients who are at highest risk and identifying the potential risk factors for oesophageal cancer development.

Tooth loss significantly influences mastication, diets, nutrition intake, aesthetics, and food choice (Adegboye et al. 2012). Evidence from observational studies have suggested that tooth loss may be associated with oesophageal and gastric cancers (Abnet et al. 2008; Hiraki et al. 2008; Patel et al. 2013; Yin et al. 2016) and oral cancer (Wang et al. 2013; Zuo et al. 2015). Although tooth loss and oesophageal cancer share common risk factors, such as alcohol and tobacco use, it is unclear if tooth loss is a 
risk indicator for oesophageal cancer. Recently, a number of epidemiological studies have been conducted to examine the association between tooth loss and susceptibility to oesophageal cancer. However, the findings were mixed and inconsistent, with some of the studies reporting positive effects (Abnet et al. 2008; Hiraki et al. 2008; Patel et al. 2013) and others failing to demonstrate a significant association (Abnet et al. 2001, 2005a; Dar et al. 2013; Guha et al. 2007; Michaud et al. 2008). Although most of the studies included a very large number of potential subjects, the number of individual cases of oesophageal cancers was very small. Given the poor prognosis of oesophageal cancer and relatively small sample size of a single study, we aimed to summarise the association between tooth loss and risk of oesophageal cancer by conducting a meta-analysis. Clarifying this relationship may emphasise the importance of considering additional preventive methods for oesophageal cancer. The study was reported following the Preferred Reporting Items for Systematic Reviews and Meta-Analyses (PRISMA) Statement criteria (Moher et al. 2009).

\section{Methods}

\section{Literature search}

To identify all potentially eligible studies, a literature search was performed in PubMed and EMBASE databases for papers published from 1966 to June 2015 without restriction to regions, publication types, or languages. To identify eligible studies, the main search employed various combinations of Medical Subject Headings $(\mathrm{MeSH})$ and non-MeSH terms: "esophageal cancer" OR "oesophageal cancer" OR "oesophageal neoplasms" OR "oesophageal squamous cell carcinoma" OR "oesophageal adenocarcinoma" and "tooth loss" OR "teeth loss". References from eligible articles were also retrieved.

\section{Eligibility criteria}

In the meta-analysis, the selected studies were considered eligible if they met the following inclusion criteria: (1) study design was either cohort, case control or cross-sectional studies; (2) the exposure was tooth loss; (3) the outcome was oesophageal cancer risk; (4) relative risk (RR) or odds ratio (OR) and hazard ratio (HR) with its $95 \%$ confidence interval (CI) (or data to calculate these) were reported. Editorial letters, historical reviews and descriptive studies, such as case reports and case series, were excluded from the study. If the included population was duplicated in more than one study, only the most comprehensive study with the largest sample size was included. Two authors (SJK and YXH) independently assessed the inclusion of all retrieved studies and resolved any disagreements through discussion or after consultation with a third author (HGL).

\section{Data extraction}

Two authors (SJK and YXH) independently extracted data from the selected studies using a standardised data extraction form. The following key points were collected: first author's surname; year of publication; study design; country; duration of follow-up; sex; total number of cases and subjects; assessment methods for tooth loss; and multiple adjusted RR, OR and HR of tooth loss and corresponding $95 \%$ CI for each category of exposure. The adjusted RR was extracted in preference to the nonadjusted RR; however, the unadjusted OR and CI were calculated when the OR was not provided. When more than one adjusted OR was reported, the ratio with the most number of adjusted variables was selected. Disagreements between reviewers regarding data extraction were resolved through discussion.

\section{Statistical analysis}

The OR with $95 \% \mathrm{CI}$ was used as the common measure across all eligible studies. Because tooth loss caused oesophageal cancer was considered a rare event, the differences among estimates of relative risk were ignored and the HR and RR were directly converted to OR. A random-effects model of the DerSimonian and Laird method was used to calculate the summary risk estimates regardless of heterogeneity (DerSimonian and Laird 1986), which incorporates both within-study and betweenstudy variabilities. Sensitivity analysis was performed to evaluate robustness and stability by sequentially omitting one study on each turn. Moreover, subgroup analysis was performed to explore the potential presence of heterogeneity and assess the influence of different inclusion criteria on the overall estimate.

The Newcastle-Ottawa Scale (NOS) was employed to evaluate the methodological quality of each study. Three major components were collected: selection of the study groups ( $0-4$ points), ascertainment for the exposure of interest in the studies ( $0-3$ points) and quality of the adjustment for confounding ( $0-2$ points). The full score was nine stars, and the high-quality study was defined as a study with $\geq 5$.

We also conducted a dose-response analysis using the method proposed by Greenland and Longnecker (1992). This method required that the distribution of cases and person-years or non-cases and risk estimates within the variance are known for at least three quantitative exposure categories. We explored a potential non-linear dose-response relationship between tooth loss and risk of oesophageal cancer using the generalised least squares for trend estimation and restricted cubic spline with four knots at 5, 35, 65 and $95 \%$ of the distribution.

Publication bias was evaluated using Begg's and Egger's tests (rank correlation and linear regression methods, 
respectively) (Begg and Mazumdar 1994; Egger et al. 1997). All statistical analyses were carried out using Stata version 13.1 (StataCorp, College Station, TX, USA).

\section{Results}

Literature search and study characteristics

A diagram showing the details of study inclusion is shown in Fig. 1. Using the outlined search strategy and selection based on the inclusion criteria, 182 studies were screened, 29 were excluded because they were duplicates and 139 were excluded based on their titles and abstracts. Fourteen full-text articles were reviewed for further assessment. One article was excluded because it was a correspondence (Conway 2009), and two articles were also excluded because the exposure was not related to tooth loss (Lee et al. 2014; Sepehr et al. 2005), three articles were subsequently excluded because the outcome was oesophageal squamous dysplasia (Dye et al. 2007; Wei et al. 2005) and upper gastrointestinal cancer (Abnet et al. 2005b). One article involved two case-control studies from central Europe and Latin America, so the article was regarded two studies (Guha et al. 2007). Finally, eight articles (nine studies) were considered eligible for inclusion in the meta-analysis (Fig. 1).

A total of eight articles (nine studies), six case-control studies (1804 cases and 5824 controls) and three cohort studies (800 oesophageal cancer cases and 106,367 participants) contributed to the analysis. The characteristics of the included case-control and cohort studies are listed in Table 1.

The eligible articles were published from 2001 to 2013. The number of oesophageal cancer patients ranged from 49 to 620 in the cohort studies and from 132 to 703 in the case-control studies. Four studies were conducted in Asia (Abnet et al. 2001, 2008; Dar et al. 2013; Hiraki

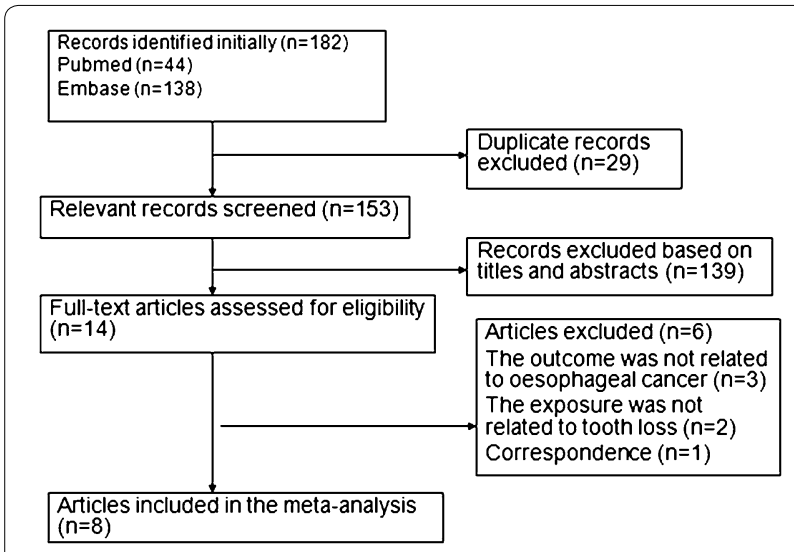

Fig. 1 Flow chart from identification of eligible studies to final inclusion et al. 2008), two in Europe (Abnet et al. 2005a; Guha et al. 2007), one in North America (Michaud et al. 2008), one in Africa (Patel et al. 2013) and one in Latin America (Guha et al. 2007). In all articles, cases were histologically, pathologically or clinically confirmed as oesophageal cancer and clearly showed the endpoint assessment of the diagnostic criteria. However, tooth loss was assessed using different strategies. Three articles used a questionnaire to classify tooth loss (Abnet et al. 2005a; Hiraki et al. 2008; Patel et al. 2013), whereas clinical examination was used as a diagnostic criteria in four articles (Abnet et al. 2001, 2008; Dar et al. 2013; Guha et al. 2007). The other one article was a self-report study (Michaud et al. 2008).

Five articles reported OR (Abnet et al. 2008; Dar et al. 2013; Guha et al. 2007; Hiraki et al. 2008; Patel et al. 2013), two reported HR (Abnet et al. 2005a; Michaud et al. 2008) and the other one reported RR (Abnet et al. 2001). One article was exclusive to men (Michaud et al. 2008), whereas the remaining studies included both men and women (Abnet et al. 2001, 2005a, 2008; Dar et al. 2013; Guha et al. 2007; Hiraki et al. 2008; Patel et al. 2013). One article did not adjust for confounding factors (Patel et al. 2013), but the other seven articles adjusted to various risk factors for oesophageal cancer, such as age, sex and education (Abnet et al. 2001, 2005a, 2008; Dar et al. 2013; Guha et al. 2007; Hiraki et al. 2008; Michaud et al. 2008). In addition, six articles controlled adjusted values, such as smoking and alcohol drinking (Abnet et al. 2001, 2008; Dar et al. 2013; Guha et al. 2007; Hiraki et al. 2008; Michaud et al. 2008).

We used NOS to evaluate the quality of the eligible studies (Table 2), in which the median NOS score was 6.5 (range of 4-8).

\section{Risk of tooth loss on oesophageal cancer events}

The meta-analysis showed that compared with the lowest category, tooth loss was associated with $53 \%$ higher rate in the highest group (OR 1.53, $95 \%$ CI 1.02-2.29), and a significant heterogeneity was detected $\left(I^{2}=72.8 \%\right.$, heterogeneity $\mathrm{P}=0.000$ ) (Fig. 2). In sensitivity analysis, the unstable results for oesophageal cancer risk was observed, which ranged from 1.29 (95\% CI 1.00 $1.67)$ with low heterogeneity $\left(I^{2}=23.3 \%, \mathrm{P}_{\text {for heterogene- }}\right.$ ity $=0.244$ ) [excluding the study by Patel et al. (2013)] to 1.67 (95\% CI 1.10-2.53) with significant heterogeneity $\left(I^{2}=72.2 \%, \mathrm{P}_{\text {for heterogeneity }}=0.001\right)$ [excluding the study by Abnet et al. (2005a)]. When stratifying the data into subgroups based on different exclusion criteria, the results are significantly inconsistent (Table 3).

\section{Dose-response meta-analysis}

Five studies (six articles) were included in the doseresponse association between tooth loss and risk of 


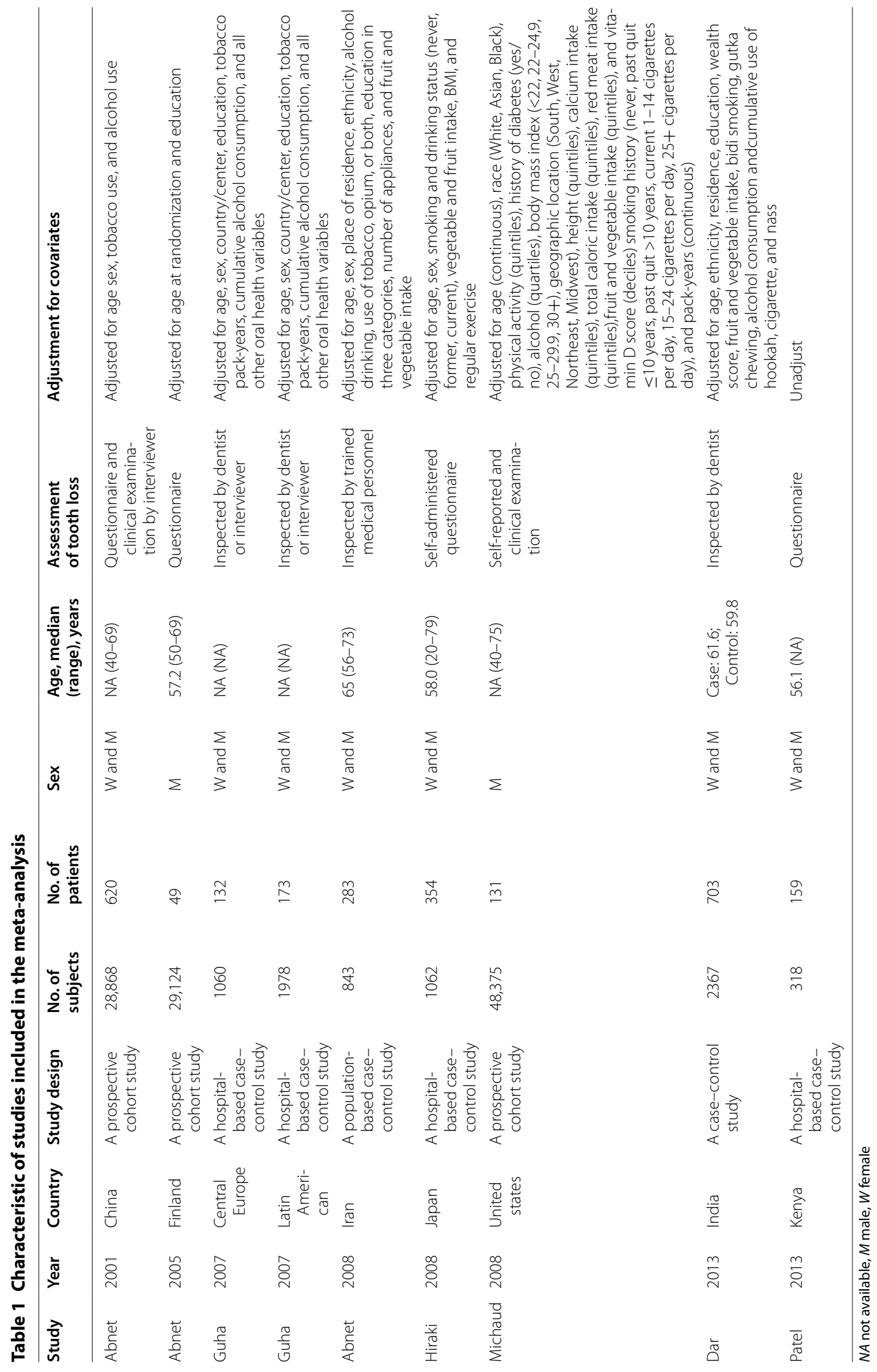


Table 2 Quality assessment of included studies based on Newcastle-Ottawa scale

\begin{tabular}{lllll}
\hline Author & Year & Selection & Comparability & Exposure \\
\hline Abnet & 2001 & 3 & 1 & 2 \\
Abnet & 2005 & 3 & 2 & 2 \\
Guha & 2007 & 3 & 2 & 3 \\
Abnet & 2008 & 3 & 1 & 2 \\
Hiraki & 2008 & 3 & 2 & 2 \\
Michaud & 2008 & 3 & 1 & 3 \\
Dar & 2013 & 3 & 1 & 2 \\
Patel & 2013 & 2 & 0 & 2 \\
\hline
\end{tabular}

oesophageal cancer, with a total of 1421 cases and 81,828 participants (Abnet et al. 2005a; Dar et al. 2013; Guha et al. 2007; Hiraki et al. 2008; Michaud et al. 2008). In dose-response analysis, the summary OR for loss of each one tooth loss increment was 1.01 (95\% CI 1.00-1.02), and no evidence of nonlinear relationship was observed (P for nonlinearity test $=0.08$; Fig. 3 ).

\section{Publication bias}

Both Begg's test and Egger's funnel plot asymmetry test (rank correlation test and regression method, respectively) in the meta-analysis indicated no significant publication bias (Begg's test, $\mathrm{P}=0.917$; Egger's test, $\mathrm{P}=0.920$; Fig. 4).

\section{Discussion}

To the best of our knowledge, this meta-analysis is the first to explore the association between tooth loss and risk of oesophageal cancer. The pooled results from the meta-analysis of nine observational studies (eight articles) using a random effects model revealed positive association between tooth loss and risk of oesophageal cancer.

Results from subgroup analyses indicated country, effect size, sample size, adjustment for smoking and alcohol drinking, quality of NOS scale, assessment of tooth loss and study design might be potential sources of heterogeneity. Despite of intrinsic limitations of observational study, some results from subgroup analyses remain notable. In subgroup analyses for study design, when we restricted the analysis to the six case-control studies and three cohort studies, the summary OR of any fracture for the highest category of tooth loss versus lowest category were 1.93 (95\% CI 1.14-3.25) and 1.02 (95\% CI 0.711.46). The combined OR for oesophageal cancer was 0.84 (95\% CI 0.47-1.52) for studies conducted in Europe, and

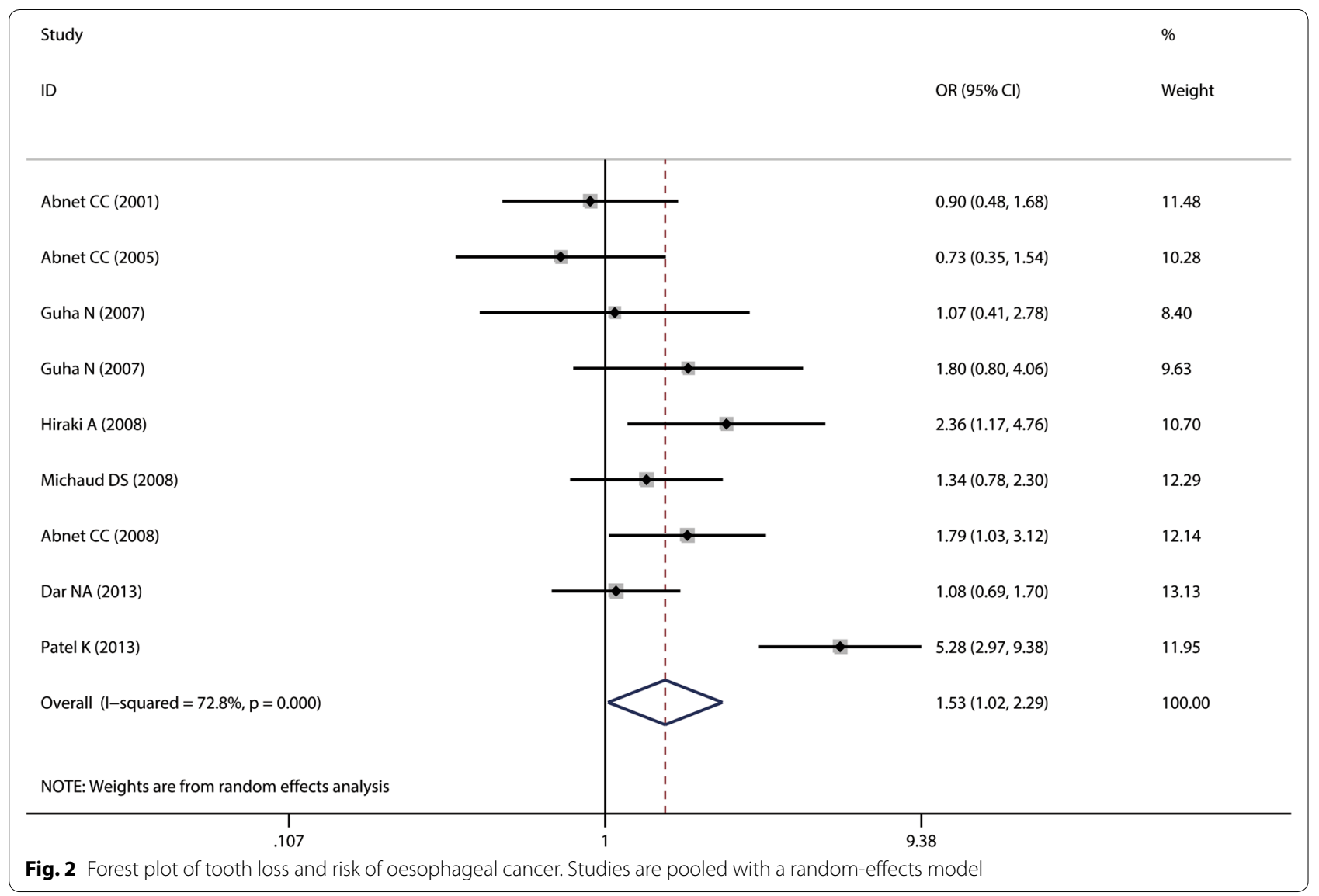


Table 3 Summary of results

\begin{tabular}{|c|c|c|c|c|c|}
\hline & Studies, N & OR (95 \% Cl) & $P$ value & $\mathrm{P}$ of heterogeneity & $I^{2}(\%)$ \\
\hline Total & 9 & $1.53(1.02-2.29)$ & 0.040 & 0.000 & 72.8 \\
\hline \multicolumn{6}{|l|}{ Country } \\
\hline Asia & 4 & $1.38(0.92-2.07)$ & 0.118 & 0.112 & 49.9 \\
\hline Europe & 2 & $0.84(0.47-1.52)$ & 0.570 & 0.536 & 0.0 \\
\hline America & 1 & $1.34(0.78-2.30)$ & 0.289 & NA & NA \\
\hline Latin America & 1 & $1.80(0.80-4.06)$ & 0.157 & NA & NA \\
\hline Africa & 1 & $5.28(2.97-9.38)$ & 0.000 & NA & NA \\
\hline \multicolumn{6}{|l|}{ Effect size } \\
\hline $\mathrm{OR}$ & 6 & $1.93(1.14-3.25)$ & 0.014 & 0.001 & 74.8 \\
\hline $\mathrm{HR}$ & 2 & $1.05(0.58-1.88)$ & 0.880 & 0.196 & 40.3 \\
\hline $\mathrm{RR}$ & 1 & $0.90(0.48-1.68)$ & 0.740 & NA & NA \\
\hline \multicolumn{6}{|l|}{ Sample size } \\
\hline Large & 5 & $1.84(0.99-3.42)$ & 0.055 & 0.000 & 83.0 \\
\hline Small & 4 & $1.19(0.84-1.71)$ & 0.329 & 0.410 & 0.0 \\
\hline \multicolumn{6}{|c|}{ Adjustment for smoking and alcohol drinking } \\
\hline Yes & 7 & $1.36(1.06-1.74)$ & 0.014 & 0.350 & 10.4 \\
\hline No & 2 & $1.99(0.29-13.85)$ & 0.486 & 0.000 & 94.1 \\
\hline \multicolumn{6}{|l|}{ NOS score } \\
\hline High & 8 & $1.29(1.00-1.67)$ & 0.053 & 0.244 & 23.3 \\
\hline Low & 1 & $5.28(2.97-9.38)$ & 0.000 & NA & NA \\
\hline \multicolumn{6}{|l|}{ Assessment of tooth loss } \\
\hline Inspected by dentists or interviewers & 5 & $1.25(0.95-1.64)$ & 0.113 & 0.413 & 0.0 \\
\hline Questionnaires & 3 & $2.13(0.69-6.58)$ & 0.190 & 0.000 & 88.3 \\
\hline Self-reported & 1 & $1.34(0.78-2.30)$ & 0.289 & NA & NA \\
\hline \multicolumn{6}{|l|}{ Study design } \\
\hline Case control study & 6 & $1.93(1.14-3.25)$ & 0.014 & 0.001 & 74.8 \\
\hline Cohort & 3 & $1.02(0.71-1.46)$ & 0.910 & 0.385 & 0.0 \\
\hline
\end{tabular}

OR odds ratio, Cl confidence interval, NA not available, Large $\geq 100$ cases, Small $<100$ cases, High NOS score of $\geq 5$, Low NOS score of $<5$

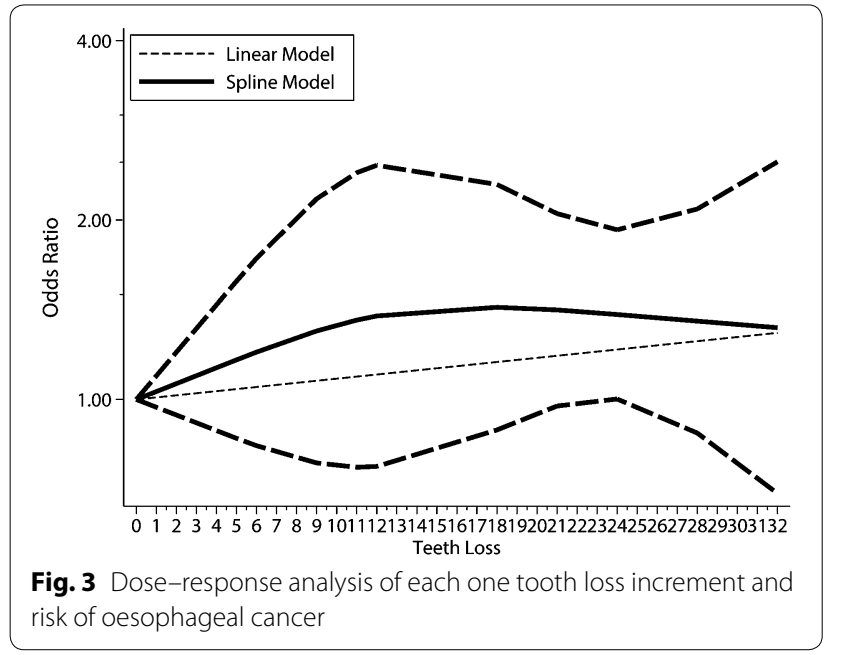

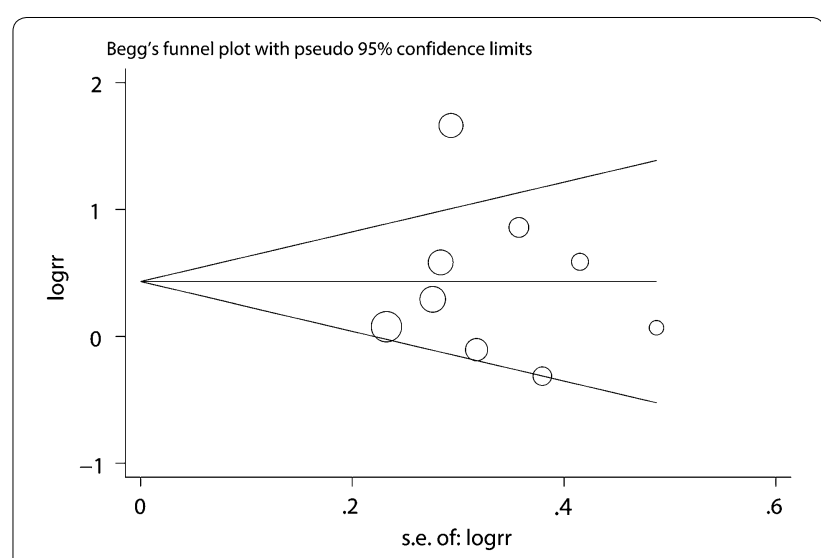

Fig. 4 Begg's funnel plot for publication bias analysis for tooth loss and risk of oesophageal cancer 
1.38 (95 \% CI 0.92-2.07) in Asia. Furthermore, smoking and alcohol consumption is considered to be significant and dependent risk factor for oesophageal cancer risk. So, we also performed subgroup analyses among studies adjusted for smoking and alcohol consumption. Only seven studies adjusted for smoking status, and the results showed tooth loss was associated with increased risk of oesophageal cancer (OR 1.36, 95 \% CI 1.06-1.74), with low heterogeneity $\left(I^{2}=10.4 \%, \mathrm{P}_{\text {for heterogeneity: }} 0.350\right)$. However, when stratified by sample size and assessment of tooth loss, a nonsignificant association was detected.

Evidence from observational studies shows that tooth loss has been associated with multiple adverse health effects including epilepsy (Karolyhazy et al. 2005), cardiovascular disease (CVD) (Joshipura et al. 1998; Lowe et al. 2003; Watt et al. 2012), cognitive impairment (Luo et al. 2015; Peres et al. 2015; Zhu et al. 2015), and cancer (Idrissi Janati et al. 2016; Yin et al. 2016). However, no definitive mechanisms were established between tooth loss and cancer (Fitzpatrick and Katz 2010; Meyer et al. 2008). Tooth loss is a marker of systemic inflammation (Buchwald et al. 2013). The scientific rationale behind the potential association is that inflammation is a major factor in both tooth loss and cancer (Coussens and Werb 2002; Karin et al. 2006; van Kempen et al. 2006). Furthermore, the oral cavity, which provides a gateway between the external environment and the esophagus/ gastrointestinal tract, functions in food ingestion and digestion. Tooth loss is also related to poorer oral hygiene (Adegboye et al. 2012; Marshall et al. 2002). Poorer oral hygiene potentially affects the gastrointestinal flora and nutritional status and may thus have implications for the development of cancer (Huang et al. 2016; Oji and Chukwuneke 2012).

This meta-analysis presents several limitations that must be considered in interpreting the results. Firstly, case-control studies have intrinsic limitations, such as selective bias and recall or memory bias. This limitation can partly explained the different results between case-control and cohort studies in the stratified analysis. Secondly, although the meta-analysis was based on a large number of participants with only nine studies included and was devoid of interventional studies, the combined estimates remained questionable. Thirdly, although we selected the highest multivariableadjusted effect estimates in the meta-analysis, we cannot neglect the effect of residual confounding factors, such as diabetes, gastroesophageal reflux, and socioeconomic status. Fourthly, a significant heterogeneity was detected. Heterogeneity among studies should not be ignored even if it is highly common in a meta-analysis. Studies included in this meta-analysis are heterogeneous in terms of different populations investigated and diagnostic criteria for tooth loss, thereby contributing to the heterogeneity in the pooled analysis. Furthermore, unstable results were observed in subgroup and sensitivity analysis, which indicated that more relevant articles are needed to further explore this association. Fifthly, tooth loss assessment varied among studies. Clinical examination was used in four articles to classify tooth loss. A questionnaire was used as diagnostic criteria of tooth loss in three articles whereas one article was self-reported. The findings are likely to be influenced by misclassification of exposure because the majority of studies employed different methods to assess and categorize tooth loss. Therefore, the results should be considered with caution because of exposure misclassification. Overall, these limitations may affect our final conclusions.

In conclusion, our meta-analysis indicates that tooth loss is a potential marker of oesophageal cancer, suggesting that people who have lost teeth should pay attention to the symptoms for oesophageal cancer. However, we can not concluded at this time that tooth loss may be a casual factor for oesophageal cancer due to significant heterogeneity among studies and mixed results between case-control studies and cohort studies. Additional large-scale and high-quality prospective studies are required to evaluate the association between tooth loss and risk of oesophageal cancer.

\section{Authors' contributions \\ JKS, GLH, XHY, SYL, YDW wrote the main manuscript text; JKS, GLH, XHY, HL, JXP prepared Figs. 1, 2, 3 and 4, JKS, GLH, YL, YDW contributed reagents/mate- rials/analysis tools. All authors read and approved the final manuscript. \\ Author details \\ ${ }^{1}$ Department of Oral and Maxillofacial Surgery, Guizhou Provincial People's Hospital, Guizhou 550002, China. ${ }^{2}$ Department of Orthodontics, Stomatology Hospital of Guiyang, Guizhou 550002, China.}

\section{Acknowledgements}

None.

Competing interests

The authors declare that they have no competing interests.

Received: 28 August 2015 Accepted: 29 June 2016

Published online: 08 July 2016

\footnotetext{
References

Abnet CC, Qiao YL, Mark SD, Dong ZW, Taylor PR, Dawsey SM (2001) Prospective study of tooth loss and incident esophageal and gastric cancers in China. Cancer Causes Control 12:847-854

Abnet CC, Kamangar F, Dawsey SM, Stolzenberg-Solomon RZ, Albanes D, Pietinen P, Virtamo J, Taylor PR (2005a) Tooth loss is associated with increased risk of gastric non-cardia adenocarcinoma in a cohort of Finnish smokers. Scand J Gastroenterol 40:681-687

Abnet CC, Qiao YL, Dawsey SM, Dong ZW, Taylor PR, Mark SD (2005b) Tooth loss is associated with increased risk of total death and death from upper gastrointestinal cancer, heart disease, and stroke in a Chinese populationbased cohort. Int J Epidemiol 34:467-474
} 
Abnet CC, Kamangar F, Islami F, Nasrollahzadeh D, Brennan P, Aghcheli K, Merat S, Pourshams A, Marjani HA, Ebadati A, Sotoudeh M, Boffetta P, Malekzadeh R, Dawsey SM (2008) Tooth loss and lack of regular oral hygiene are associated with higher risk of esophageal squamous cell carcinoma. Cancer Epidemiol Biomark Prev 17:3062-3068

Adegboye AR, Twetman S, Christensen LB, Heitmann BL (2012) Intake of dairy calcium and tooth loss among adult Danish men and women. Nutrition 28:779-784

Begg CB, Mazumdar M (1994) Operating characteristics of a rank correlation test for publication bias. Biometrics 50:1088-1101

Buchwald S, Kocher T, Biffar R, Harb A, Holtfreter B, Meisel P (2013) Tooth loss and periodontitis by socio-economic status and inflammation in a longitudinal population-based study. J Clin Periodontol 40:203-211

Castellsague X, Munoz N (1997) Esophageal cancer: its epidemiology, risk factors and prevention. Gastroenterol Hepatol 20:231-238

Conway D (2009) Oral health, mouthwashes and cancer-what is the story? Evid Based Dent 10:6-7

Coussens LM, Werb Z (2002) Inflammation and cancer. Nature 420:860-867

Dar NA, Islami F, Bhat GA, Shah IA, Makhdoomi MA, lqbal B, Rafiq R, Lone MM, Abnet CC, Boffetta P (2013) Poor oral hygiene and risk of esophageal squamous cell carcinoma in Kashmir. Br J Cancer 109:1367-1372

DerSimonian R, Laird N (1986) Meta-analysis in clinical trials. Control Clin Trials 7:177-188

Dye BA, Wang R, Lashley R, Wei W, Abnet CC, Wang G, Dawsey SM, Cong W, Roth MJ, Li X, Qiao Y (2007) Using NHANES oral health examination protocols as part of an esophageal cancer screening study conducted in a high-risk region of China. BMC Oral Health 7:10

Egger M, Davey Smith G, Schneider M, Minder C (1997) Bias in meta-analysis detected by a simple, graphical test. BMJ 315:629-634

Engel LS, Chow WH, Vaughan TL, Gammon MD, Risch HA, Stanford JL, Schoenberg JB, Mayne ST, Dubrow R, Rotterdam H, West AB, Blaser M, Blot W Gail MH, Fraumeni JF Jr (2003) Population attributable risks of esophageal and gastric cancers. J Natl Cancer Inst 95:1404-1413

Enzinger PC, Mayer RJ (2003) Esophageal cancer. N Engl J Med 349:2241-2252

Fitzpatrick SG, Katz J (2010) The association between periodontal disease and cancer: a review of the literature. J Dent 38:83-95

Greenland S, Longnecker MP (1992) Methods for trend estimation from summarized dose-response data, with applications to meta-analysis. Am J Epidemiol 135:1301-1309

Guha N, Boffetta P, Wunsch Filho V, Eluf Neto J, Shangina O, Zaridze D, Curado MP, Koifman S, Matos E, Menezes A, Szeszenia-Dabrowska N, Fernandez L, Mates D, Daudt AW, Lissowska J, Dikshit R, Brennan P (2007) Oral health and risk of squamous cell carcinoma of the head and neck and esophagus: results of two multicentric case-control studies. Am J Epidemiol 166:1159-1173

Hiraki A, Matsuo K, Suzuki T, Kawase T, Tajima K (2008) Teeth loss and risk of cancer at 14 common sites in Japanese. Cancer Epidemiol Biomark Prev 17:1222-1227

Huang J, Roosaar A, Axell T, Ye W (2016) A prospective cohort study on poor oral hygiene and pancreatic cancer risk. Int J Cancer 138:340-347

Idrissi Janati A, Durand R, Karp I, Voyer R, Latulippe JF, Emami E (2016) Association between oral conditions and colorectal cancer: a literature review and synthesis. Rev Epidemiol Sante Publique 64:113-119

Joshipura KJ, Douglass CW, Willett WC (1998) Possible explanations for the tooth loss and cardiovascular disease relationship. Ann Periodontol 3:175-183

Karin M, Lawrence T, Nizet V (2006) Innate immunity gone awry: linking microbial infections to chronic inflammation and cancer. Cell 124:823-835

Karolyhazy K, Kivovics P, Fejerdy P, Aranyi Z (2005) Prosthodontic status and recommended care of patients with epilepsy. J Prosthet Dent 93:177-182

Lee YL, Hu HY, Yang NP, Chou P, Chu D (2014) Dental prophylaxis decreases the risk of esophageal cancer in males; a nationwide population-based study in Taiwan. PLoS One 9:e109444

Lowe G, Woodward M, Rumley A, Morrison C, Tunstall-Pedoe H, Stephen K (2003) Total tooth loss and prevalent cardiovascular disease in men and women: possible roles of citrus fruit consumption, vitamin C, and inflammatory and thrombotic variables. J Clin Epidemiol 56:694-700
Luo J, Wu B, Zhao Q, Guo Q, Meng H, Yu L, Zheng L, Hong Z, Ding D (2015) Association between tooth loss and cognitive function among 3063 Chinese older adults: a community-based study. PLoS One 10:e0120986

Marshall TA, Warren JJ, Hand JS, Xie XJ, Stumbo PJ (2002) Oral health, nutrient intake and dietary quality in the very old. J Am Dent Assoc 133:1369-1379

Meyer MS, Joshipura K, Giovannucci E, Michaud DS (2008) A review of the relationship between tooth loss, periodontal disease, and cancer. Cancer Causes Control 19:895-907

Michaud DS, Liu Y, Meyer M, Giovannucci E, Joshipura K (2008) Periodontal disease, tooth loss, and cancer risk in male health professionals: a prospective cohort study. Lancet Oncol 9:550-558

Moher D, Liberati A, Tetzlaff J, Altman DG, Group P (2009) Preferred reporting items for systematic reviews and meta-analyses: the PRISMA statement. BMJ 339:b2535

Napier KJ, Scheerer M, Misra S (2014) Esophageal cancer: a Review of epidemiology, pathogenesis, staging workup and treatment modalities. World J Gastrointest Oncol 6:112-120

Oji C, Chukwuneke F (2012) Poor oral hygiene may be the sole cause of oral cancer. J Maxillofac Oral Surg 11:379-383

Patel K, Wakhisi J, Mining S, Mwangi A, Patel R (2013) Esophageal cancer, the topmost cancer at MTRH in the Rift Valley, Kenya, and its potential risk factors. ISRN Oncol 2013:503249. doi:10.1155/2013/503249

Peng Q, Chen H, Huo JR (2016) Alcohol consumption and corresponding factors: a novel perspective on the risk factors of esophageal cancer. Oncol Lett 11:3231-3239

Pennathur A, Gibson MK, Jobe BA, Luketich JD (2013) Oesophageal carcinoma. Lancet 381:400-412

Peres MA, Bastos JL, Watt RG, Xavier AJ, Barbato PR, D'Orsi E (2015) Tooth loss is associated with severe cognitive impairment among older people: findings from a population-based study in Brazil. Aging Ment Health 19:876-884

Sepehr A, Kamangar F, Fahimi S, Saidi F, Abnet CC, Dawsey SM (2005) Poor oral health as a risk factor for esophageal squamous dysplasia in northeastern Iran. Anticancer Res 25:543-546

Siegel RL, Miller KD, Jemal A (2016) Cancer statistics, 2016. CA Cancer J Clin 66:7-30

van Kempen LC, de Visser KE, Coussens LM (2006) Inflammation, proteases and cancer. Eur J Cancer 42:728-734

Wang JB, Fan JH, Liang H, Li J, Xiao HJ, Wei WQ, Dawsey SM, Qiao YL, Boffetta P (2012) Attributable causes of esophageal cancer incidence and mortality in China. PLoS One 7:e42281

Wang RS, Hu XY, Gu WJ, Hu Z, Wei B (2013) Tooth loss and risk of head and neck cancer: a meta-analysis. PLoS One 8:e71122

Watt RG, Tsakos G, de Oliveira C, Hamer M (2012) Tooth loss and cardiovascular disease mortality risk-results from the Scottish Health Survey. PLoS One 7:e30797

Wei WQ, Abnet CC, Lu N, Roth MJ, Wang GQ, Dye BA, Dong ZW, Taylor PR, Albert P, Qiao YL, Dawsey SM (2005) Risk factors for oesophageal squamous dysplasia in adult inhabitants of a high risk region of China. Gut 54:759-763

Yin XH, Wang YD, Luo H, Zhao K, Huang GL, Luo SY, Peng JX, Song JK (2016) Association between Tooth Loss and Gastric Cancer: a meta-analysis of observational studies. PLoS One 11:e0149653

Zhu J, Li X, Zhu F, Chen L, Zhang C, McGrath C, He F, Xiao Y, Jin L (2015) Multiple tooth loss is associated with vascular cognitive impairment in subjects with acute ischemic stroke. J Periodontal Res 50:683-688

Zuo C, Zhu Y, Wang X, Zeng X, Huang C (2015) Tooth loss and risk of oral squamous cell carcinoma in Chinese Han population. Int J Clin Exp Med 8:21893-21897 